\title{
Subquantum-Well Influence on Carrier Dynamics in High Efficiency DUV Dislocation-Free AIGaN/AIGaN-Based Multiple Quantum Wells
}

\author{
Idris A. Ajia, Dhiafallah Almalawi, Yi Lu, Sergei Lopatin, Xiaohang Li, Zhiqiang Liu, and Iman S. Roqan*
}

Cite This: ACS Photonics 2020, 7, 1667-1675

Read Online

ABSTRACT: We explore the effect of the subwell centers and related carrier dynamics mechanisms in dislocation-free DUV AlGaN/AlGaN multiple quantum wells (MQWs) homoepitaxially grown on an AlN substrate. Cross-sectional imaging and energydispersive X-ray compositional analyses using scanning transmission electron microscopy (STEM) reveal epitaxial layers of very high crystalline quality, as well as ultrathin Al-rich subquantum barrier and subwell layers at the interface between the wells and the barriers. Carrier dynamic analyses studied by power- and

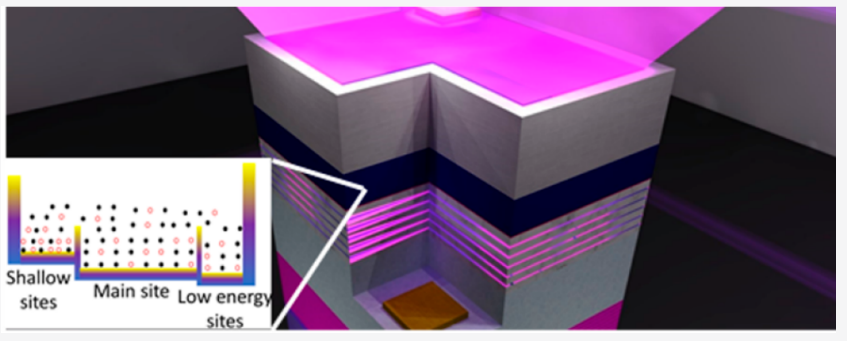
temperature-dependent time-resolved and time-integrated photoluminescence (PL) and PL excitation measurements, as well as numerical simulations, reveal the carrier repopulation mechanisms between the MQWs and subwell sites. This advanced analysis shows that the subwell/sub-barrier structure results in additional exciton localization centers, enhancing the internal quantum efficiency via staggered carrier repopulation into the MQWs to reach a maximum of $\sim 83 \%$ internal quantum efficiency, which remains high at high injected carrier densities in the droop region. Both experimental and numerical simulation results show that the slight efficiency droop can be due to Auger recombination, counteracted by a simultaneous increase in radiative recombination processes at high power density, demonstrating the role of the subwells/sub-barriers in efficiency enhancement.

KEYWORDS: subquantum-barrier, homoepitaxy, AlN substrate, compositional pulling, dislocation-free, deep UV, ultrafast lasers, carrier dynamics

$\mathrm{D}$ eep ultraviolet (DUV) light-emitting devices have a variety of useful applications, including germicidal irradiation, phototherapy, water, air, and food sterilization, charge management in space-based sensors and photocatalysis, among others. ${ }^{1-4}$ Although $\mathrm{Hg}$ vapor-based lamps have traditionally served as UV light source for these applications, their effectiveness is severely limited due to bulkiness, slow response time, fragility, temperature sensitivity, high power consumption, and potential environmental hazards. ${ }^{5-8}$ As such, AlGaN-based light-emitting diodes (LEDs) are considered a viable alternative by both researchers and industry practitioners, as their use eliminates the aforementioned drawbacks, while offering the advantage of wide spectral tunability for a multitude of applications. ${ }^{9}$ While AlGaN-based DUV LEDs have been achieved in a research context, their industrial success has been limited, primarily due to poor external quantum efficiency $(<15 \%) .{ }^{3,10}$ Recently, extensive work on AlGaN-based DUV LEDs has been conducted to enhance epitaxial growth quality and improve device internal quantum efficiency (IQE) by reducing the threading dislocation (TD) density arising from the lattice mismatch between the grown materials and substrates. Many growth enhancement techniques have proven successful in improving the IQE of AlGaN-based DUV LEDs. For instance, a thick AlN template on sapphire has been used to reduce TD density cleaving through the epitaxial layers, thereby increasing the overall efficiency of the III-nitride-based devices. ${ }^{11-13}$ Strainedlayer superlattices (SLSs) have also been employed to improve epitaxial morphology of the resulting devices. ${ }^{14-16}$ Recently, by combining SLS with a nanopatterned sapphire substrate, the IQE value of the DUV AlGaN/AlGaN multiple quantum well (MQW) has been enhanced. ${ }^{17}$ Although, the use of homoepitaxial (native AlN) substrates renders these structure-enhancing techniques redundant by ensuring that devices are free from TDs, such DUV devices still suffer from low external efficiency, accompanied by a droop at high carrier densities. ${ }^{18,19}$ However, other structural enhancements, such as spontaneous subwell formation, that can improve the device efficiency have not yet been studied. Particularly, no research on carrier dynamics has been conducted to investigate the effect of subwells/sub-barriers surrounding MQW structures

Received: December 23, 2019

Published: May 26, 2020 
on the DUV device efficiency and the contributions of the nonradiative and Auger recombination.

In this work, we reveal the carrier dynamics of a DUV $\mathrm{AlGaN} / \mathrm{AlGaN} \mathrm{MQW}$ structure grown on native AlN substrate. The advanced structural investigations reveal the presence of subwell and sub-barrier layers sandwiching the MQWs in the active layer. Optical analyses show the effect of these understudied structural aberrations on the carrier dynamics, optical efficiency and efficiency droop inside the active regions.

\section{SAMPLE GROWTH AND EXPERIMENTAL TECHNIQUES}

The DUV structure was prepared by metalorganic chemical vapor deposition (MOCVD). Trimethyl-aluminum (TMAl), trimethyl-gallium (TMGa), and $\mathrm{NH}_{3}$ were used as the precursor reactants. A $400 \mathrm{~nm}$ thick AlN buffer layer was initially regrown on a $1 \mathrm{in}$. thick (0001) AlN substrate at 1250 ${ }^{\circ} \mathrm{C}$, followed by a $20 \times \mathrm{Al}_{0.4} \mathrm{Ga}_{0.6} \mathrm{~N} / \mathrm{AlN}$ SLSs layer to enhance the quality of the active layer. (The chemical composition of SLS structure was estimated by energy dispersive X-ray spectroscopy (EDX) that are attached to high-resolution transmission electron microscopy (HR-TEM), as shown in Figure S1 in Supporting Information.) Subsequently, a $1 \mu \mathrm{m}$ thick $\mathrm{n}-\mathrm{AlGaN}$ layer was grown, followed by a nominal fiveperiod $\mathrm{Al}_{0.4} \mathrm{Ga}_{0.6} \mathrm{~N} / \mathrm{Al}_{0.5} \mathrm{Ga}_{0.5} \mathrm{~N}(3 \mathrm{~nm} / 12 \mathrm{~nm})$ multiple quantum well (MQW) active layer, which was capped by a $30 \mathrm{~nm}$ thick $\mathrm{p}-\mathrm{Al}_{0.65} \mathrm{Ga}_{0.35} \mathrm{~N}$ electron blocking layer (EBL).

DUV MQW structure lamellae were prepared for high-angle annular dark field-scanning transmission electron microscopy images (HAADF-STEM) using an FEI Helios focused ion beam (FIB) and scanning electron microscope (SEM). The HAADF-STEM images were acquired using a Cs-probe corrected FEI Titan HR-TEM system, operated at an acceleration voltage of $300 \mathrm{kV}$. EDX maps were acquired using the system attached to FEI Titan HR-TEM. For photoluminescence (PL) measurements, the third harmonic line $(250 \mathrm{~nm})$ of an ultrafast (150 fs) Ti:sapphire pulsed laser $(76 \mathrm{MHz})$ served as the excitation source. The emitted signal was subsequently spectrally resolved using a SpectraPro 2300 spectrograph fitted with a grating with $150 \mathrm{gr} / \mathrm{mm}$ groove density and a blaze of $300 \mathrm{~nm}$. A Hamamatsu C9300 chargecoupled device (CCD) camera detected the emission arising from the sample. The excitation source was focused to a beam diameter of $\sim 60 \mu \mathrm{m}$ for power-dependent PL measurements to enhance the power density incident on the sample. For temperature-dependent time integrated (TIPL) and timeresolved PL (TRPL) measurements, the sample was enclosed in a Janis ST-100 closed cycle cryostat under high vacuum conditions. A LakeShore 335 temperature controller was employed to vary the sample holder's temperature within the 5-300 $\mathrm{K}$ range. To temporally resolve the detected signal, the excitation pulse frequency was first reduced to $2 \mathrm{MHz}$ using A.P.E PulseSelect pulse picker, after which a Hamamatsu C5680 streak camera with a slow sweep unit, fitted between the CCD and the spectrograph, was activated. Each sweep signal was synchronized with the reduced pulse frequency of the excitation source. The detected signal was subsequently acquired in photon counting mode. Room temperature (RT) PL excitation (PLE) was carried out using FLS1000 Edinburgh Instruments spectrometer attached to a $1000 \mathrm{~W}$ Xe lamp. APSYS, a commercial TCAD software developed by Crosslight Inc., was used to numerically simulate an LED device with a similar active region as the DUV structure. The software selfconsistently calculates Poisson's equation, the current continuity equation, the carrier transport equations and the quantum mechanical wave equations of the device, ${ }^{20}$ from which information, such as the current-dependent IQE, position dependence of the bandgap energy, carrier concentration in the active region, and carrier recombination rates can be extracted.

\section{RESULTS AND DISCUSSION}

Figure 1a shows the cross-sectional STEM image of the full sample structure. The thicknesses of the various pre-MQW

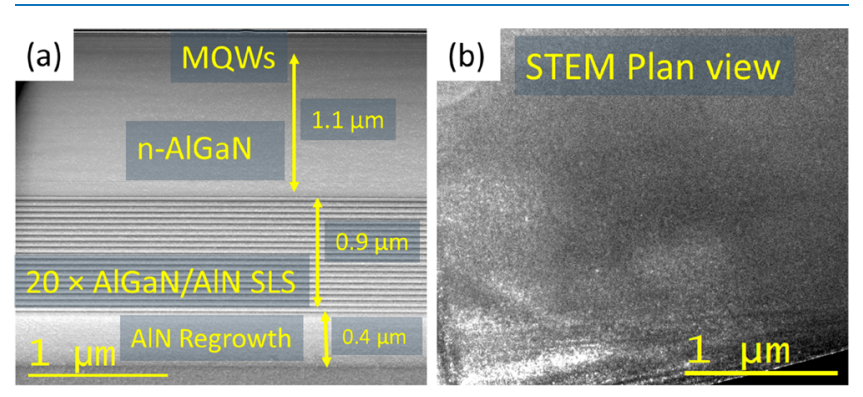

Figure 1. (a) STEM cross-sectional image of the AlGaN/AlGaN LED structure grown of AlN substrate; (b) $2.7 \times 2.7 \mu \mathrm{m}$ plan-view STEM image of the sample.

layers indicated in the STEM image are typical of similar structures grown on sapphire. ${ }^{21,22}$ As expected, no TD defects are observed, as confirmed in the HR-STEM plan-view images of the structure shown in Figure $1 \mathrm{~b}$, where no evidence of spots that are characteristic of TD defects is found. ${ }^{23-25}$ This is not surprising, considering that the MQWs are preceded by an AlN buffer layer that was homoepitaxially grown on an AlN substrate characterized by very low TD density (typically $<10^{4}$ $\left.\mathrm{cm}^{-2}\right) .{ }^{26}$ In addition, the SLS structure further mitigates the propagation of TDs to subsequent layers. ${ }^{17,22,27}$

A magnified image of the MQWs and EBL layers is shown in Figure $2 \mathrm{a}$, further confirming that the MQWs are well-defined, with no apparent interruptions from TDs. Figure $2 b$ shows a magnified STEM image of a single quantum well, indicating presence of ultrathin layers sandwiching the MQWs. To further identify these layers, EDX was carried out during HRTEM measurements to study the chemical composition of Ga, $\mathrm{Al}$, and $\mathrm{N}$ content, as shown in Figure 2c. The chemical composition profile shown in Figure $2 \mathrm{~d}$ reveals that all MQWs are neighbored by a shallow subquantum well (sub-QW) on one side, which has a lower Ga concentration (indicated by a black arrow in Figure 2d) compared to that in the main MQWs, and is separated by an ultrathin barrier from the main MQW $(<1 \mathrm{~nm})$. On the other edge of the MQWs, an additional Al-rich layer forms, providing further ultrathin barrier (sub-QB) of $<1 \mathrm{~nm}$ width and characterized by a slightly higher Al concentration compared to the quantum barrier (QB), as indicated by red arrow in Figure $2 \mathrm{~d}$. This distribution was confirmed for all MQWs, as shown in Figure $2 \mathrm{~d}$. Such sub-QB structures have previously been observed and were attributed to a "compositional pulling" effect, where the smaller of the group-III atoms $(\mathrm{Al})$ preferentially drift toward the barrier layer that exerts a compressive strain on the well during MOCVD growth. ${ }^{28-32}$

Figure 3a shows the PL spectra of the structure at 5 and 290 $\mathrm{K}(\mathrm{RT})$, indicating that, at $5 \mathrm{~K}$, the main MQW PL peak is 

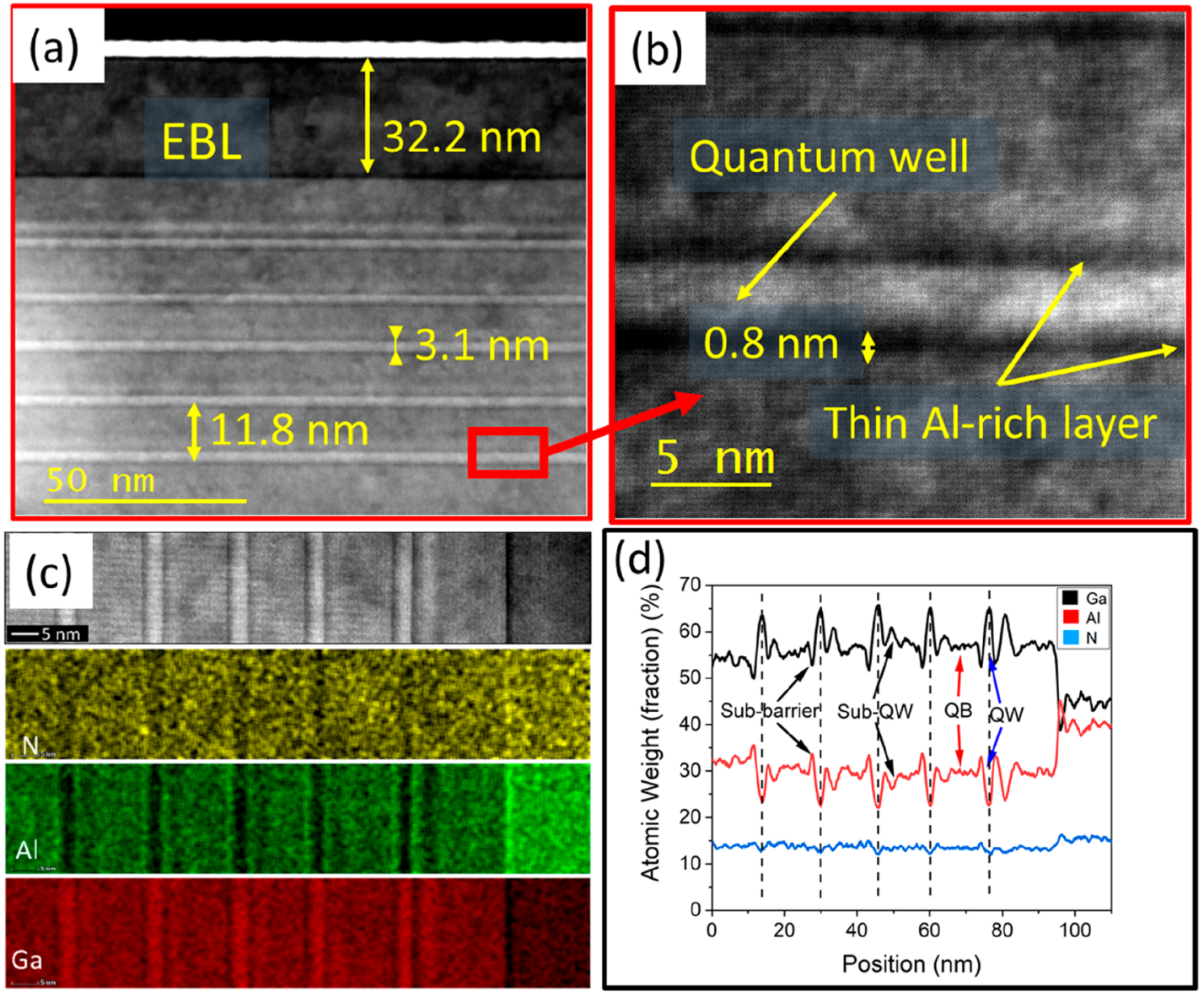

Figure 2. (a) Magnified STEM image of the MQW region showing the thickness of wells, barriers, and the electron blocking layer; (b) Magnified STEM image showing additional Al-rich layers at the interface between the well and the barrier; (c) EDX cross-sectional maps of the elemental composition of the active region; (d) Atomic weight distribution profiles of $\mathrm{Ga}$, $\mathrm{Al}$, and $\mathrm{N}$ in the active region of the DUV structure.

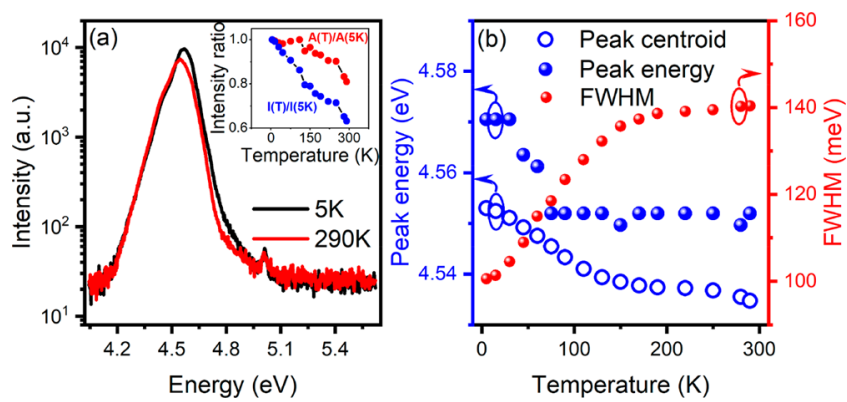

Figure 3. (a) PL spectra of the MQW structure at 5 and $290 \mathrm{~K}$. Inset: Integrated intensity ratio, $\mathrm{IQE}=A(T) / A(5 \mathrm{~K})$ (linked red circles), and peak intensity ratio, $I(T) / I(5 \mathrm{~K})$ (linked green circles); (b) Temperature dependence of the peak energy (blue spheres), fwhm (red spheres), and the PL centroid.

located at $4.57 \mathrm{eV}$ and is slightly red-shifted to $4.55 \mathrm{eV}$ at RT. However, a slight shoulder at $4.44 \mathrm{eV}$ is visible on the lower energy side of the spectrum. The ratio of integrated intensity at $\mathrm{RT}$ and at low temperature, $A(290 \mathrm{~K}) / A(5 \mathrm{~K})$, was found to be $81 \%$, confirming high optical quality. The temperaturedependent ratios of the integrated intensity, $A(T) / A(5 \mathrm{~K})$ (linked red circles), and peak intensity, $I(T) / I(5 \mathrm{~K})$ (linked blue circles), are plotted against temperature $(T)$ in the inset of Figure 3a. Note that, as no TD propagation to MQWs was observed, $A(T) / A(5 \mathrm{~K})$ is a qualitative representation of the IQE values of the MQW emission, based on the assumption that, at cryogenic temperatures, carriers lack the thermal energy required for their capture by nonradiative centers around TD defect sites, according to Rashba's treatment. ${ }^{33-36}$ At any rate, the occurrence of TD density is expected to be very low in $\mathrm{Al}$-rich $\mathrm{AlGaN}$ epitaxial layers are grown on $\mathrm{AlN}$ substrates (as the structure is grown on a lattice-matched substrate). ${ }^{37,38}$ Thus, in such a structure, the increased distance between nonradiative centers reduces the probability of nonradiative recombination. ${ }^{39}$ Therefore, high IQE values are expected of structures exhibiting high crystalline quality free from TDs. On the other hand, as the temperature increases, there is a notable divergence between the slopes of the integrated intensity ratio, $A(T) / A(5 \mathrm{~K})$, and the peak intensity ratio, $I(T) / I(5 \mathrm{~K})$. This temperature-dependent difference indicates that carrier redistribution (affected by the potential differences between the MQWs and sub-QWs) plays a significant role in the recombination dynamics of the structure.

To elucidate the carrier distribution phenomenon further, the PL centroid (defined as the weighted average peak of all the photon energies represented in the PL emission spectrum ranging, in this case, from 4.30 to $4.80 \mathrm{eV}),{ }^{40}$ as well as the full width at half-maximum (fwhm) of the emission spectrum and peak energy, are plotted (in Figure $3 b$ ) as functions of temperature. At $5 \mathrm{~K}$, the peak energy is located at $\sim 4.57 \mathrm{eV}$, whereas the peak abruptly redshifts to $\sim 4.55 \mathrm{eV}$ in the $30-75$ $\mathrm{K}$ temperature range, after which the peak energy remains unchanged as temperature increases to $290 \mathrm{~K}$. Given that the 

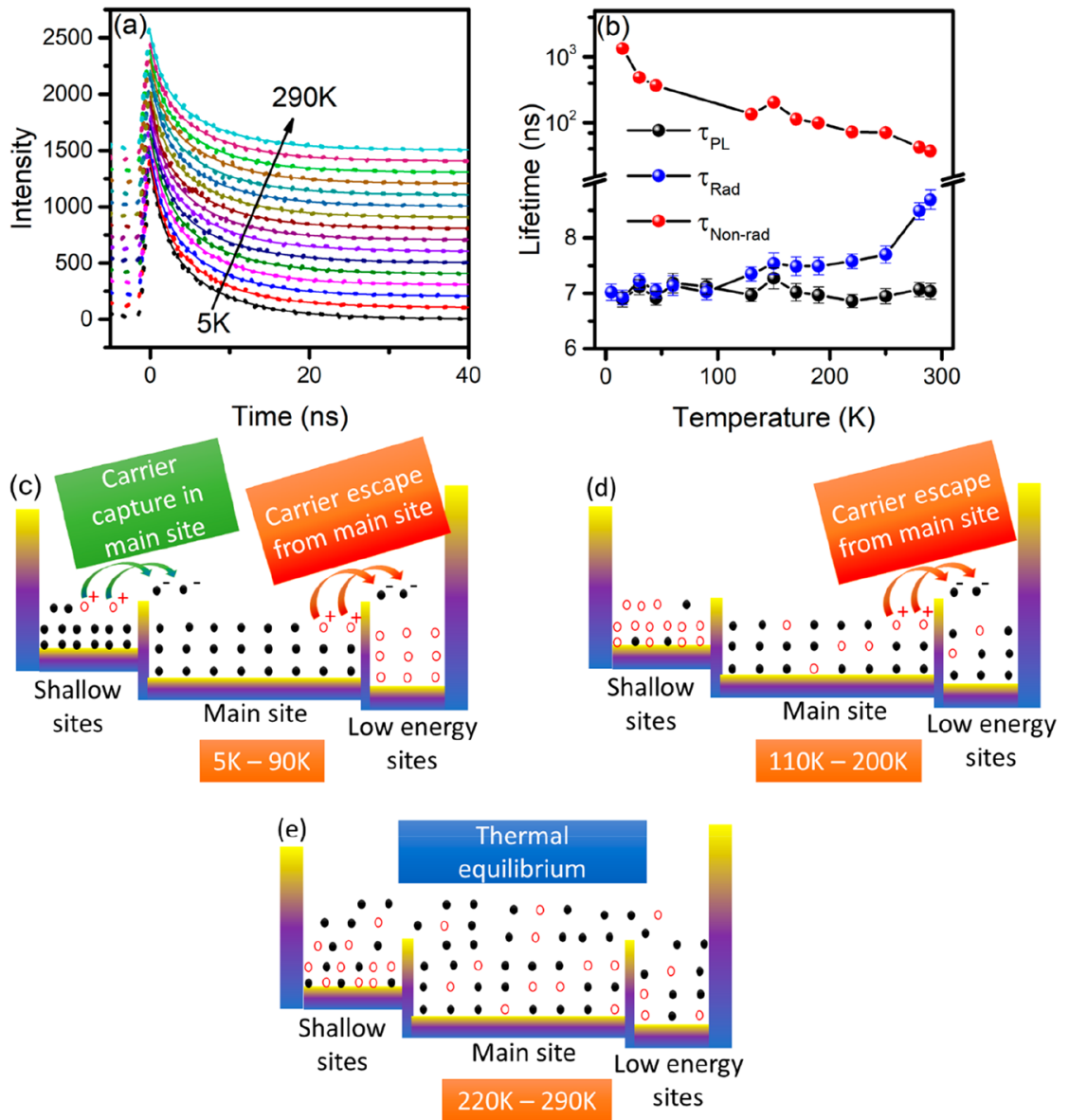

Figure 4. (a) TRPL lifetime responses (dots) at different temperatures, along with their corresponding fittings (lines). (b) PL (black spheres), radiative (blue spheres), and nonradiative (red spheres) lifetimes as a function of temperature. Illustrations of the carrier dynamics in the MQW structure at (c) low, (d) intermediate, and (e) high temperatures.

peak energy is virtually independent of temperature above 75 $\mathrm{K}$, a direct relationship between bandgap shrinkage and temperature increase due to thermally induced dilation of the crystal lattice can be ruled out. ${ }^{41,42}$ Indeed, this independence can be attributed to strong carrier confinement within the MQWs, with the peak energy centered at $\sim 4.55 \mathrm{eV}$. Such strong confinement in the MQWs is aided by the Al-rich sub-QB layers, which act as additional high-energy barriers in the structure. On the other hand, the initial redshift in peak energy as the temperature increases from 30 to $75 \mathrm{~K}$ is attributed to rapid thermionic repopulation of randomly distributed carriers from weakly confined states in the shallow sub-QWs at very low temperatures to the main recombination sites within the MQWs through tunneling and hopping, as the ultrathin $\mathrm{QB}$ between MQW and sub-QW is only $<1 \mathrm{~nm}$ thick. $^{43,44}$

The fwhm plot shown in Figure $3 \mathrm{~b}$ reveals that the line width broadens as the temperature increases, starting from $\sim 100 \mathrm{meV}$ at $5 \mathrm{~K}$, and reaching a saturation value of $\sim 140$ $\mathrm{meV}$ in the 190-290 K range, confirming our hypothesis. This broadening indicates that, after settling at the main MQW recombination sites, thermal delocalization causes a proportion of the carriers to migrate to other recombination centers in the vicinity of the localized recombination site at $4.55 \mathrm{eV}$. However, since the peak energy remains practically constant above $75 \mathrm{~K}$ (which is lower than the saturation temperature of the fwhm), the PL centroid energy plot as a function of temperature was studied to determine the carrier flow direction. The PL centroid energy plot should be sensitive to the energy state of the migrating carriers since it is a weighted average peak of all the photon energies represented in the PL spectrum. As shown in Figure $3 \mathrm{~b}$, in the $5-100 \mathrm{~K}$ range, the centroid energy decreases as the temperature increases. Here, saturation is achieved at $190 \mathrm{~K}$, following an opposite trend to that shown in the fwhm plot. This observation suggests that, aided by thermal excitation, a small fraction of migrating carriers has red-shifted $(4.535 \mathrm{eV})$ to energy states located below $4.55 \mathrm{eV}$.

To further understand the carrier dynamics of the MQW structure, it was subjected to temperature-dependent TRPL measurements, as indicated in Figure 4a. The lifetime values were obtained by fitting the experimental data (dots) to the biexponential lifetime decay model (lines; see Section S2 in Supporting Information for details) due to different recombination centers. ${ }^{45}$ Figure $4 \mathrm{~b}$ shows the PL lifetime $\tau_{\mathrm{PL}}$ (black spheres) as a function of temperature. Interestingly, no obvious $\tau_{\mathrm{PL}}$ dependence on temperature is observed, confirming strong dominance of radiative recombination. It is known that the radiative recombination rate is independent of temperature.

In order to study the specific roles of radiative and nonradiative processes and the carrier dynamics within the MQWs, the radiative $\left(\tau_{\mathrm{Rad}}\right)$ and nonradiative $\left(\tau_{\text {Non-rad }}\right)$ lifetimes of the carriers with respect to temperature were extracted from the PL lifetimes, as shown in Figure $4 b .^{46-48}$ 

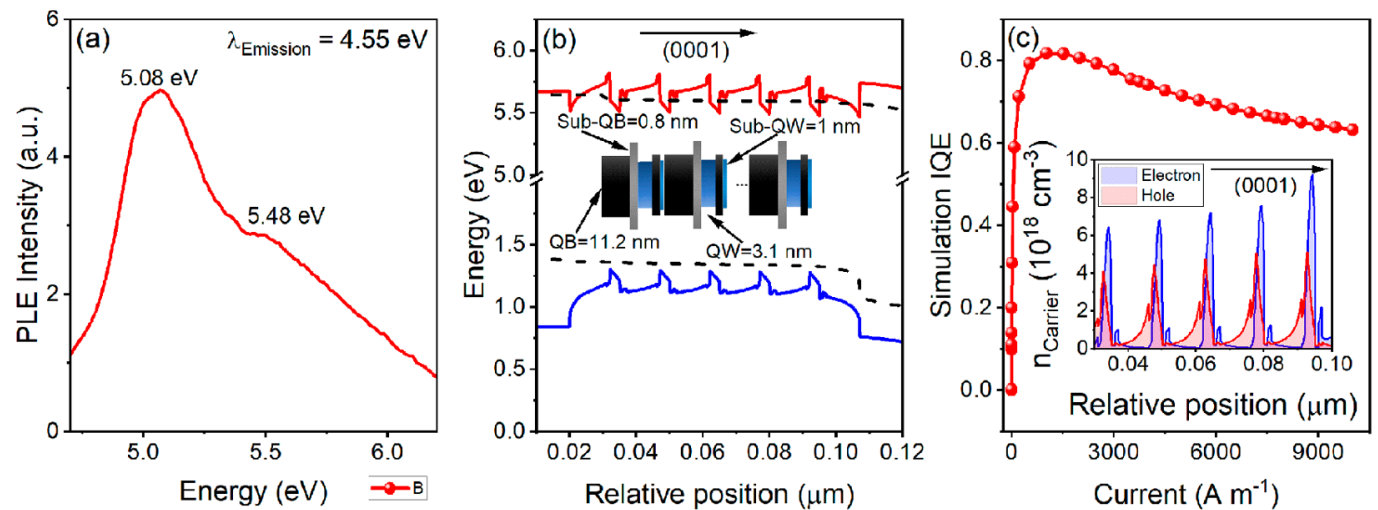

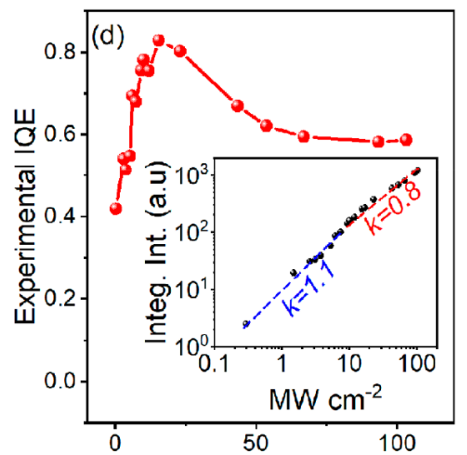

Pulse Power Density $\left(\mathrm{MW} \mathrm{cm}^{-2}\right)$
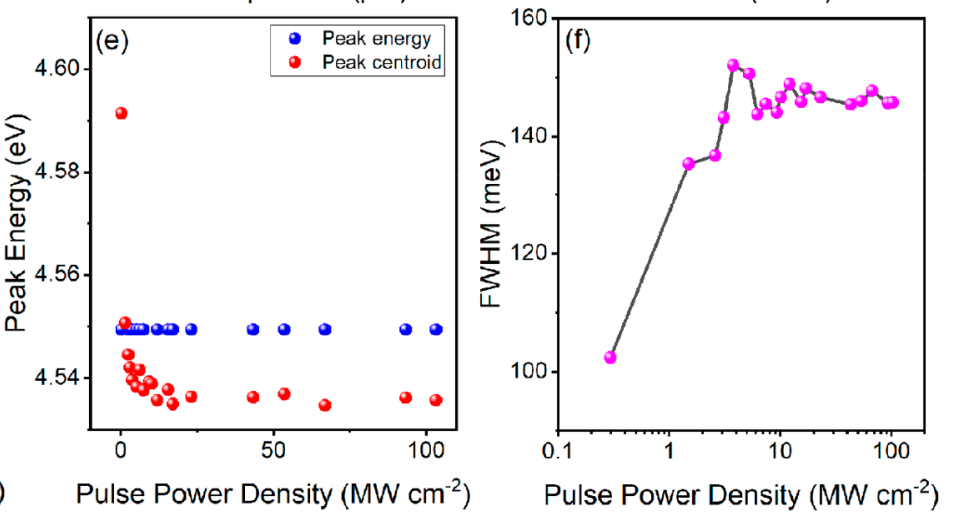

Figure 5. (a) PLE spectrum taken at the main emission band-edge energy $(4.55 \mathrm{eV})$. (b) Band diagram of the active region of the simulated DUV LED device. (c) Simulated IQE of the active region of the DUV LED device. Inset: Simulated electron and hole concentration under $10000 \mathrm{~A} / \mathrm{m}$ current injection. (d) Power-dependent IQE at RT using the SRH method. Inset: log-log plot of the integrated intensity of the MQWs with respect to pulse power density. (e) Power-dependent peak energy and peak centroid. (f) Power-dependent fwhm in log scale.

No crossover point has been observed for the studied temperature range, as the radiative lifetime is dominant. The obtained findings revealed that $\tau_{\text {Non-rad }}$ declines monotonically from $1300 \mathrm{~ns}$ at $15 \mathrm{~K}$ to $40 \mathrm{~ns}$ at $290 \mathrm{~K}$, as shown in Figure $4 \mathrm{~b}$. While this represents a significant reduction in the $\tau_{\text {Non-rad }}$ value within the temperature range of interest, it has negligible effect on the overall carrier lifetime of the MQWs $\left(\tau_{\mathrm{PL}}\right)$. On the other hand, $\tau_{\text {Rad }}$ of $7 \mathrm{~ns}$ was obtained at $5 \mathrm{~K}$ and remains constant until $90 \mathrm{~K}$. This temperature range $(5-90 \mathrm{~K})$ coincides with the range at which an abrupt redshift in peak energy occurs, as shown in Figure $3 \mathrm{~b}$. Since the significant carrier migration to the localized recombination sites $(\sim 4.55$ $\mathrm{eV}$ ) observed in this range $(5-90 \mathrm{~K})$ has no notable influence on the radiative efficiency $(\mathrm{IQE}=A(T) / \mathrm{A}(5 \mathrm{~K})$ ) of the MQWs, as indicated in the inset of Figure $3 \mathrm{a}$, it is proposed that the constant $\tau_{\mathrm{PL}}$ is due to a dynamic equilibrium between the rate of carriers (from shallow sites in the sub-QW) captured by the localized sites within the main MQW (at 4.55 $\mathrm{eV}$ ) and carrier escape from the main sites of MQW to other lower energy states, as shown in Figure 4c. These lower-energy sites can be due to lateral inhomogeneities in the MQWs. ${ }^{21,22}$

At intermediate temperatures $(110-200 \mathrm{~K})$, there is a very gradual increase in $\tau_{\text {Rad }}$ from 7 to $7.7 \mathrm{~ns}$. At this point, most of the carriers have been transferred from the shallow sites to the main localization sites at $4.55 \mathrm{eV}$. Consequently, the rate of carrier escape from the main sites to the lower-energy sites now exceeds the rate of capture, as shown in Figure 4d. This carrier repopulation mechanism causes a gradual decrease in carrier density within the main sites, leading to an increase in $\tau_{\text {Rad }}$.
At high temperatures $(T>220 \mathrm{~K})$, the carriers approach thermal equilibrium, due to which they become randomly distributed across the main MQW, sub-QW, and lower-energy sites, as shown in Figure 4e. Thus, the resulting emission will be due to transitions from all these sites. Notably, it is within this temperature range that the fwhm approaches its saturation value of $140 \mathrm{meV}$, indicating that, at this point, most of the states have reached thermal equilibrium and there is no net carrier migration, as illustrated in Figure 4e. Figure 5a shows that the RT PLE spectrum obtained at the MQW emission peak $(4.55 \mathrm{eV})$ presented in Figure $3 \mathrm{a}$, which indicates that such emission originates from two sources, the MQW band edge (located at $5.08 \mathrm{eV}$ ) and an additional higher-energy edge located at $5.48 \mathrm{eV}$. The higher-energy edge can be due to the transitions from the sub-QW. This result confirms our hypothesis that the carriers approach thermal equilibrium and are distributed across all sites. However, the contribution from the main MQW states predominates due to the higher density of states compared to the other two types (sub-QW and lower energy states).

We simulated an LED device with similar sub-QW, sub-QB, $M Q W$ and $Q B$ in its active region (see Section $S 3$ in Supporting Information and Figure S3) to gain insight into the distribution of electrons and holes in the active regions, as well as to estimate the recombination efficiency of the active region (MQW, sub-QW, and sub-QB, as shown in Figure S4). The simulated LED was subjected to an injection current of 10000 $\mathrm{A} \mathrm{m}^{-1}$, while the nonradiative lifetime, radiative recombination coefficient and Auger recombination coefficients were set at 8 $\mathrm{ns}, 4 \times 10^{-8} \mathrm{~cm}^{3} / \mathrm{s}$ and $3 \times 10^{-30} \mathrm{~cm}^{6} / \mathrm{s}$, respectively, consistent with commonly made assumptions. ${ }^{18,49,50}$ The band 
diagram of the LED's active region is shown in Figure 5b, which clearly reveals the presence of the sub-QW and sub-QB regions. In Figure 5c, the current-dependent IQE of the simulated LED is shown. The IQE peaks at $81 \%$ (which is in line with IQE estimated by temperature-dependent $\mathrm{PL}$ ) when the injection current is set to $1500 \mathrm{~A} \mathrm{~m}^{-1}$, after which we witness the onset of Auger recombination. The inset of Figure $5 \mathrm{c}$ shows the position dependence of electron and hole concentrations in the active region. It is noteworthy that, while the simulation showed that most of the carriers are concentrated within the MQWs, as expected, it indicated that still a small fraction of carriers are inside the sub-QW layers, supporting the observation previously made by PLE.

We carried out power-dependent PL spectroscopy at RT, which allowed us in order to systematically study the IQE values and efficiency droop of the MQW emission, as well as the effect of nonradiative recombination, using the ShockleyRead-Hall (SRH) method, where the excitation pulse powerdependent carrier generation rate, $G(P)$, is given by ${ }^{51}$

$$
G(P)=A n(P)+B n(P)^{2}+C n(P)^{3}
$$

where $A n(P)$ represents the Shockley-Read-Hall (SRH) nonradiative recombination rate, $B n(P)^{2}$ is the radiative recombination rate, and $C n(P)^{3}$ is the Auger nonradiative recombination rate. The integrated PL intensity can be defined as $I_{\mathrm{PL}}=\beta B n^{2}$ (here, we have adopted $A_{\mathrm{PL}}(T)=I_{\mathrm{PL}}$ to differentiate between $A$ of the SRH coefficient and the integrated intensity $A(T)$, and $\beta$ represents a constant determined by the total collection efficiency from the active region volume). Hence, eq 1 can be rewritten in terms of $I_{\mathrm{PL}}$ yielding

$$
G(P)=A \sqrt{\frac{I_{\mathrm{PL}}(P)}{\beta B}}+\frac{I_{\mathrm{PL}}(P)}{\beta}+C\left(\frac{I_{\mathrm{PL}}(P)}{\beta B}\right)^{3 / 2}
$$

This equation shows the three regimes as a relation between carrier generation rate $(G)$ and PL intensity $\left(I_{\mathrm{PL}}\right)$, given by $I_{\mathrm{PL}}$ $\propto G^{k}$, whereby $k=2$, applies to a nonradiative SRH recombination regime at low carrier injection; $k=2 / 3$ relates to the Auger recombination regime at high carrier injection; and $k=1$ holds where a radiative recombination regime dominates. $^{52}$

According to the SRH method described by eq 1 , the powerdependent IQE can be derived with the following equation, as explained elsewhere: ${ }^{45,53}$

$$
\operatorname{IQE}(P)=\frac{B n(P)^{2}}{G(P)}
$$

The curve depicting IQE as a function of excitation pulse power density is presented in Figure $5 d$, where an increase in IQE with power density can be observed up to a maximum value of $\sim 83 \%$ at $15 \mathrm{MW} \mathrm{cm} \mathrm{cm}^{-2}$. This value is comparable with both the thermal IQE determined by the integrated $\mathrm{PL}$ intensity ratio, $A(290 \mathrm{~K}) / A(5 \mathrm{~K})$ and the numerically simulated IQE result. This value is also in accordance with the IQE reported previously for AlGaN/AlGaN MQWs grown on native AlN substrate. ${ }^{18}$ However, beyond this point, a slight efficiency droop that saturates at $\sim 60 \%$ was noted, indicating the significant role of the exciton localization in the sub-QW states.

In general, four possible mechanisms have been proposed as the cause of efficiency droop in III-nitride quantum wells, namely, Auger recombination, ${ }^{54,55}$ carrier overflow/delocalization from the quantum well into the p-region, which is not an efficient radiative recombination center, ${ }^{56,57}$ defect-assisted tunneling, ${ }^{58,59}$ and current density assisted defect recombination. ${ }^{60}$ However, we rule out the latter two mechanisms as the main cause of the droop in our MQW structure, given that it is practically TD-free. To determine if the source of this droop was carrier overflow, we plotted the peak energy and centroid energy against power density, as shown in Figure 5e. Typically, efficiency droop due to carrier overflow should be accompanied by a blueshift and broadening of the peak energy, since this implies migration to, and subsequent recombination of the carriers at, higher less localized energy states. ${ }^{45,61}$ However, no such blueshift was observed in the emission produced by the MQW structure under investigation; rather, the peak energy remained constant, as indicated by the blue-colored curve in Figure 5e. Furthermore, at a power density of $\sim 10 \mathrm{MW} \mathrm{cm}{ }^{-2}$, the peak centroid energy red-shifted from $4.59 \mathrm{eV}$ and settled at $4.53 \mathrm{eV}$, implying that the carriers had migrated to lower energy states. This peak shift can be attributed to the shift of the dominant recombination center from the high energy subQW states (at $4.59 \mathrm{eV}$ ) at low power densities to the main MQW states $(4.53-4.55 \mathrm{eV})$.

To elucidate our findings further, we observe that the power dependence of the fwhm of the PL spectra, plotted in Figure $5 f$, initially broadens from $\sim 102 \mathrm{meV}$ as the power density increases, and rapidly saturates at $\sim 140 \mathrm{meV}$ (when the carrier density reaches $\sim 10 \mathrm{MW} \mathrm{cm}^{-2}$ ). It is noteworthy that the onset of the efficiency droop effect coincides with that of the fwhm saturation, suggesting that the peak broadening is not linked to the droop effect. This result seems to rule out carrier overflow as the possible mechanism underlying the efficiency droop. As such, Auger recombination remains the most plausible loss mechanism in this TD-free MQW structure at high power densities. ${ }^{62}$ Nonetheless, it is worth noting that, as droop saturation occurred at a relatively high IQE $(\sim 60 \%)$, the radiative recombination processes effectively counteracted the droop mechanism. This counteraction process may have been aided specifically by the concurrent migration of carriers to the highly confined low energy MQW states $(\sim 4.55 \mathrm{eV})$ at high power densities.

To confirm the origin of the droop, the $\log I_{\mathrm{PL}}$ versus $\log G^{k}$ correlation for regions before and after the droop was analyzed, as shown in the inset of Figure 5d. According to eqs 1 and 2 for the injected carrier density range below the droop region $(\leq 15$ MW $\mathrm{cm}^{-2}$ ), $k=\sim 1.1$ was obtained, confirming that the emission is significantly dominated by radiative recombination with negligible nonradiative recombination. On the other hand, $k=\sim 0.8$ was obtained at carrier densities characterizing both droop and saturation regions $\left(>15 \mathrm{MW} \mathrm{cm}^{-2}\right)$, which exceeds $2 / 3$, indicating that the recombination process is governed by majority radiative recombination competing with minor Auger recombination process. This confirms that the enhanced radiative recombination with minor Auger recombination stems from localized exciton in the sub-QWs. The numerical simulation confirms the contribution of SRH and Auger recombination under low and high carrier injection modes, respectively (as shown in Figure S4 in Supporting Information). The analysis reported in this work can be applied to any semiconductor nanostructure system. 


\section{CONCLUSIONS}

An $\mathrm{AlGaN} / \mathrm{AlGaN}$ LED structure was homoepitaxially grown on a (0001) AlN substrate. HR-STEM cross-sectional and plan-view images revealed pseudomorphic, highly crystalline epilayers that are free from TD defects. The STEM and EDX measurements confirmed the presence of additional ultrathin sub-QBs and sub-QWs, caused by compositional pulling, which play significant roles in the carrier repopulation dynamics and the exciton localization, enhancing the efficiency and radiative recombination. Temperature- and power-dependent PL and TRPL, as well as result from numerical simulation results, confirmed that the quantum wells are highly efficient recombination centers, with the IQE > 83\%. Even at RT, carrier repopulation from the sub-QWs into the QWs suppressed nonradiative processes, leading to significant improvement in IQE, while suppressing the onset of efficiency droop ( $60 \%)$. This slight droop, due to Auger recombination, competes with the simultaneous increase in radiative recombination processes. This work enhances the current understanding of the role of sub-QBs/sub-QWs effect in AlGaN/AlGaN MQW structures, which adds a potential degree of control in the quest for highly efficient DUV LED growth.

\section{ASSOCIATED CONTENT}

\section{SI Supporting Information}

The Supporting Information is available free of charge at https://pubs.acs.org/doi/10.1021/acsphotonics.9b01814.

EDX atomic composition profile of $\mathrm{Al}_{0.4} \mathrm{Ga}_{0.6} \mathrm{~N} / \mathrm{AlN}$ superlattices layer, method for extracting $\mathrm{PL}$ carrier lifetime, information about DUV LED numerical simulation parameters, and additional analyses (PDF)

\section{AUTHOR INFORMATION}

\section{Corresponding Author}

Iman S. Roqan - King Abdullah University of Science and Technology (KAUST), Physical Science and Engineering (PSE) Division, Thuwal, Saudi Arabia; 1 orcid.org/0000-00017442-4330; Email: iman.roqan@kaust.edu.sa

\section{Authors}

Idris A. Ajia - King Abdullah University of Science and Technology (KAUST), Physical Science and Engineering (PSE) Division, Thuwal, Saudi Arabia; 다. orcid.org/0000-00033156-4426

Dhiafallah Almalawi - King Abdullah University of Science and Technology (KAUST), Physical Science and Engineering (PSE) Division, Thuwal, Saudi Arabia; Physics Department, Faculty of Science, Taif University, 21974 Taif, Saudi Arabia

Yi Lu - King Abdullah University of Science and Technology (KAUST), Computer, Electrical and Mathematical Sciences and Engineering (CEMSE) Division, Thuwal, Saudi Arabia

Sergei Lopatin - King Abdullah University of Science and Technology (KAUST), Imaging and Characterization Core Laboratory, Thuwal, Saudi Arabia; 이이이.org/0000-00033916-3803

Xiaohang Li - King Abdullah University of Science and Technology (KAUST), Computer, Electrical and Mathematical Sciences and Engineering (CEMSE) Division, Thuwal, Saudi

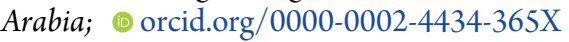

Zhiqiang Liu - Development Center, Institute of Semiconductors, Chinese Academy of Sciences, Beijing, China; (1) orcid.org/ 0000-0002-5341-0463

Complete contact information is available at:

https://pubs.acs.org/10.1021/acsphotonics.9b01814

\section{Author Contributions}

All authors have given approval to the final version of the manuscript.

Notes

The authors declare no competing financial interest.

\section{ACKNOWLEDGMENTS}

The research reported in this publication was supported by funding from King Abdullah University of Science and Technology (KAUST), using the base funding (BAS/1/ 1319-01-01).

\section{REFERENCES}

(1) Kneissl, M.; Kolbe, T.; Chua, C.; Kueller, V.; Lobo, N.; Stellmach, J.; Knauer, A.; Rodriguez, H.; Einfeldt, S.; Yang, Z.; Johnson, N. M.; Weyers, M. Advances in group III-nitride-based deep UV light-emitting diode technology. Semicond. Sci. Technol. 2011, 26, No. 014036.

(2) Khan, A.; Balakrishnan, K.; Katona, T. Ultraviolet light-emitting diodes based on group three nitrides. Nat. Photonics 2008, 2, 77-84.

(3) Wu, F.; Sun, H.; Ajia, I. A.; Roqan, I. S.; Zhang, D.; Dai, J.; Chen, C.; Feng, Z. C.; Li, X. Significant internal quantum efficiency enhancement of $\mathrm{GaN} / \mathrm{AlGaN}$ multiple quantum wells emitting at $350 \mathrm{~nm}$ via step quantum well structure design. J. Phys. D: Appl. Phys. 2017, 50, 245101.

(4) Mitra, S.; Aravindh, A.; Das, G.; Pak, Y.; Ajia, I.; Loganathan, K.; Di Fabrizio, E.; Roqan, I. S. High-performance solar-blind flexible deep-UV photodetectors based on quantum dots synthesized by femtosecond-laser ablation. Nano Energy 2018, 48, 551-559.

(5) Kim, D. Y.; Park, J. H.; Lee, J. W.; Hwang, S.; Oh, S. J.; Kim, J.; Sone, C.; Schubert, E. F.; Kim, J. K. Overcoming the fundamental light-extraction efficiency limitations of deep ultraviolet light-emitting diodes by utilizing transverse-magnetic-dominant emission. Light: Sci. Appl. 2015, 4, No. e263.

(6) Taniyasu, Y.; Kasu, M.; Makimoto, T. An aluminium nitride light-emitting diode with a wavelength of 210 nanometres. Nature 2006, 441, 325-328.

(7) Shur, M. S.; Gaska, R. Deep-Ultraviolet Light-Emitting Diodes. IEEE Trans. Electron Devices 2010, 57, 12-25.

(8) Sun, K.-X.; Allard, B.; Buchman, S.; Williams, S.; Byer, R. L LED deep UV source for charge management of gravitational reference sensors. Classical Quantum Gravity 2006, 23, S141.

(9) Matsuoka, T. Current status of GaN and related compounds as wide-gap semiconductors. J. Cryst. Growth 1992, 124, 433-438.

(10) Hirayama, H.; Maeda, N.; Fujikawa, S.; Toyoda, S.; Kamata, N. Recent progress and future prospects of AlGaN-based high-efficiency deep-ultraviolet light-emitting diodes. Jpn. J. Appl. Phys. 2014, 53, 100209.

(11) Sakai, M.; Ishikawa, H.; Egawa, T.; Jimbo, T.; Umeno, M.; Shibata, T.; Asai, K.; Sumiya, S.; Kuraoka, Y.; Tanaka, M.; Oda, O. High-quality GaN growth on AlN/sapphire templates by MOVPE. In Compound Semiconductors 2001; Arakawa, Y., Hirayama, Y., Kishino, K., Yamaguchi, H., Eds. Taylor and Francis, 2002; pp 783-788.

(12) Egawa, T.; Ohmura, H.; Ishikawa, H.; Jimbo, T. Demonstration of an InGaN-based light-emitting diode on an AlN/sapphire template by metalorganic chemical vapor deposition. Appl. Phys. Lett. 2002, 81, 292-294.

(13) Kuwano, N.; Tsuruda, T.; Kida, Y.; Miyake, H.; Hiramatsu, K.; Shibata, T. TEM analysis of threading dislocations in crack-free 
AlxGa1-xN grown on an $\mathrm{AlN}(0001)$ template. Phys. Status Solidi $C$ 2003, 0, 2444-2447.

(14) Sun, W.; Adivarahan, V.; Shatalov, M.; Lee, Y.; Wu, S.; Yang, J.; Zhang, J.; Khan, M. A. Continuous Wave Milliwatt Power AlGaN Light Emitting Diodes at $280 \mathrm{~nm}$. Jpn. J. Appl. Phys. 2004, 43, L1419.

(15) Tian, Y.; Yan, J.; Zhang, Y.; Chen, X.; Guo, Y.; Cong, P.; Sun, L.; Wang, Q.; Guo, E.; Wei, X.; Wang, J.; Li, J. Stimulated emission at $288 \mathrm{~nm}$ from silicon-doped AlGaN-based multiple-quantum-well laser. Opt. Express 2015, 23, 11334-11340.

(16) Dong, P.; Yan, J.; Wang, J.; Zhang, Y.; Geng, C.; Wei, T.; Cong, P.; Zhang, Y.; Zeng, J.; Tian, Y.; Sun, L.; Yan, Q.; Li, J.; Fan, S.; Qin, Z. 282-nm AlGaN-based deep ultraviolet light-emitting diodes with improved performance on nano-patterned sapphire substrates. Appl. Phys. Lett. 2013, 102, 241113.

(17) Wang, T.-Y.; Tasi, C.-T.; Lin, C.-F.; Wuu, D.-S. 85\% internal quantum efficiency of $280 \mathrm{~nm}$ AlGaN multiple quantum wells by defect engineering. Sci. Rep. 2017, 7, 14422.

(18) Bryan, Z.; Bryan, I.; Xie, J.; Mita, S.; Sitar, Z.; Collazo, R. High internal quantum efficiency in $\mathrm{AlGaN}$ multiple quantum wells grown on bulk AlN substrates. Appl. Phys. Lett. 2015, 106, 142107.

(19) Inoue, S.-i.; Tamari, N.; Taniguchi, M. $150 \mathrm{~mW}$ deepultraviolet light-emitting diodes with large-area AlN nanophotonic light-extraction structure emitting at $265 \mathrm{~nm}$. Appl. Phys. Lett. 2017, $110,141106$.

(20) Kuo, Y.-K.; Yen, S.-H.; Wang, Y.-W. Simulation of Deep Ultraviolet Light-Emitting Diodes; SPIE, 2007; Vol. 6669.

(21) Ajia, I. A.; Edwards, P. R.; Liu, Z.; Yan, J. C.; Martin, R. W.; Roqan, I. S. Excitonic localization in AlN-rich AlxGa1-xN/AlyGa1yN multi-quantum-well grain boundaries. Appl. Phys. Lett. 2014, 105, 122111.

(22) Peng, M. Z.; Guo, L. W.; Zhang, J.; Zhu, X. L.; Yu, N. S.; Yan, J. F.; Liu, H. H.; Jia, H. Q.; Chen, H.; Zhou, J. M. Reducing dislocations of Al-rich AlGaN by combining AlN buffer and AlN/Al0.8Ga0.2N superlattices. J. Cryst. Growth 2008, 310, 1088-1092.

(23) Arslan, E.; Ozturk, M. K.; Duygulu, Ö.; Kaya, A. A.; Ozcelik, S.; Ozbay, E. The influence of nitridation time on the structural properties of $\mathrm{GaN}$ grown on $\mathrm{Si}$ (111) substrate. Appl. Phys. A: Mater. Sci. Process. 2009, 94, 73-82.

(24) Ivanov, S. V.; Nechaev, D. V.; Sitnikova, A. A.; Ratnikov, V. V.; Yagovkina, M. A.; Rzheutskii, N. V.; Lutsenko, E. V.; Jmerik, V. N. Plasma-assisted molecular beam epitaxy of $\mathrm{Al}(\mathrm{Ga}) \mathrm{N}$ layers and quantum well structures for optically pumped mid-UV lasers on cAl2O3. Semicond. Sci. Technol. 2014, 29, No. 084008.

(25) Ajia, I. A.; Yamashita, Y.; Lorenz, K.; Muhammed, M. M.; Spasevski, L.; Almalawi, D.; Xu, J.; Iizuka, K.; Morishima, Y.; Anjum, D. H.; Wei, N.; Martin, R. W.; Kuramata, A.; Roqan, I. S. GaN/ AlGaN Multiple Quantum Wells Grown on Transparent and Conductive (-201)-Oriented $\beta$-Ga2O3 Substrate for UV Vertical Light Emitting Devices. Appl. Phys. Lett. 2018, 113, No. 082102.

(26) Zhou, T. Y.; Raghothamachar, B.; Wu, F. Z.; Dalmau, R.; Moody, B.; Craft, S.; Schlesser, R.; Dudley, M.; Sitar, Z. Characterization of Threading Dislocations in PVT-Grown AlN Substrates via $\mathrm{x}$-Ray Topography and Ray Tracing Simulation. J. Electron. Mater. 2014, 43, 838-842.

(27) Zhang, J. P.; Wang, H. M.; Gaevski, M. E.; Chen, C. Q.; Fareed, Q.; Yang, J. W.; Simin, G.; Khan, M. A. Crack-free thick AlGaN grown on sapphire using AlN/AlGaN superlattices for strain management. Appl. Phys. Lett. 2002, 80, 3542-3544.

(28) Tsai, Y.-L.; Wang, C.-L.; Lin, P.-H.; Liao, W.-T.; Gong, J.-R. Observation of compositional pulling phenomenon in AlxGal$\mathrm{xN}(0.4<\mathrm{x}<1.0)$ films grown on (0001) sapphire substrates. Appl. Phys. Lett. 2003, 82, 31-33.

(29) Lin, H. Y.; Chen, Y. F.; Lin, T. Y.; Shih, C. F.; Liu, K. S.; Chen, N. C. Direct evidence of compositional pulling effect in AlxGa1-xN epilayers. J. Cryst. Growth 2006, 290, 225-228.

(30) Li, X.; Sundaram, S.; Disseix, P.; Le Gac, G.; Bouchoule, S.; Patriarche, G.; Réveret, F.; Leymarie, J.; El Gmili, Y.; Moudakir, T.; Genty, F.; Salvestrini, J. P.; Dupuis, R. D.; Voss, P. L.; Ougazzaden, A.
AlGaN-based MQWs grown on a thick relaxed AlGaN buffer on AlN templates emitting at $285 \mathrm{~nm}$. Opt. Mater. Express 2015, 5, 380-392. (31) Liu, B.; Zhang, R.; Zheng, J. G.; Ji, X. L.; Fu, D. Y.; Xie, Z. L.; Chen, D. J.; Chen, P.; Jiang, R. L.; Zheng, Y. D. Composition pulling effect and strain relief mechanism in AlGaN/AlN distributed Bragg reflectors. Appl. Phys. Lett. 2011, 98, 261916.

(32) Li, X.-H.; Kao, T.-T.; Satter, M. M.; Wei, Y. O.; Wang, S.; Xie, H.; Shen, S.-C.; Yoder, P. D.; Fischer, A. M.; Ponce, F. A.; Detchprohm, T.; Dupuis, R. D. Demonstration of transverse-magnetic deep-ultraviolet stimulated emission from AlGaN multiple-quantumwell lasers grown on a sapphire substrate. Appl. Phys. Lett. 2015, 106, No. 041115.

(33) Rashba, E. I. Giant Oscillator-Strengths Associated with Exciton Complexes (Review). Sov. Phys. Semicond. 1975, 8, 807-816.

(34) Brandt, O.; Ringling, J.; Ploog, K. H.; Wunsche, H. J.; Henneberger, F. Temperature dependence of the radiative lifetime in GaN. Phys. Rev. B: Condens. Matter Mater. Phys. 1998, 58, 1597715980.

(35) Iida, D.; Fadil, A.; Chen, Y.; Ou, Y.; Kopylov, O.; Iwaya, M.; Takeuchi, T.; Kamiyama, S.; Akasaki, I.; Ou, H. Internal quantum efficiency enhancement of $\mathrm{GaInN} / \mathrm{GaN}$ quantum-well structures using Ag nanoparticles. AIP Adv. 2015, 5, No. 097169.

(36) Alfaraj, N.; Mitra, S.; Wu, F.; Ajia, I. A.; Janjua, B.; Prabaswara, A.; Aljefri, R. A.; Sun, H.; Khee Ng, T.; Ooi, B. S.; Roqan, I. S.; Li, X. Photoinduced entropy of InGaN/GaN p-i-n double-heterostructure nanowires. Appl. Phys. Lett. 2017, 110, 161110.

(37) Kida, Y.; Shibata, T.; Miyake, H.; Hiramatsu, K. Metalorganic Vapor Phase Epitaxy Growth and Study of Stress in AlGaN Using Epitaxial AlN as Underlying Layer. Jpn. J. Appl. Phys. 2003, 42, L572L574.

(38) Li, D.; Jiang, K.; Sun, X.; Guo, C. AlGaN photonics: recent advances in materials and ultraviolet devices. Adv. Opt. Photonics 2018, 10, 43-110.

(39) Hirayama, H., Growth Techniques of AlN/AlGaN and Development of High-Efficiency Deep-Ultraviolet Light-Emitting Diodes. In III-Nitride Ultraviolet Emitters: Technology and Applications, Kneissl, M., Rass, J., Eds.; Springer International Publishing: Cham, 2016; pp 75-113.

(40) Weisstein, E. W. Wolfram MathWorld. Function Centroid. http://mathworld.wolfram.com/FunctionCentroid.html (accessed March 20, 2018).

(41) Varshni, Y. P. Temperature dependence of the energy gap in Semiconductors. Physica 1967, 34, 149-154.

(42) Flemban, T. H.; Sequeira, M. C.; Zhang, Z.; Venkatesh, S.; Alves, E.; Lorenz, K.; Roqan, I. S. Identifying the influence of the intrinsic defects in Gd-doped $\mathrm{ZnO}$ thin-films. J. Appl. Phys. 2016, 119, No. 065301.

(43) Liu, W.; Zhao, D. G.; Jiang, D. S.; Chen, P.; Liu, Z. S.; Zhu, J. J.; Shi, M.; Zhao, D. M.; Li, X.; Liu, J. P.; Zhang, S. M.; Wang, H.; Yang, H.; Zhang, Y. T.; Du, G. T. Temperature dependence of photoluminescence spectra for green light emission from InGaN/ GaN multiple wells. Opt. Express 2015, 23, 15935-15943.

(44) Cho, Y. H.; Gainer, G. H.; Fischer, A. J.; Song, J. J.; Keller, S.; Mishra, U. K.; DenBaars, S. P. S-shaped" temperature-dependent emission shift and carrier dynamics in InGaN/GaN multiple quantum wells. Appl. Phys. Lett. 1998, 73, 1370.

(45) Ajia, I. A.; Edwards, P. R.; Pak, Y.; Belekov, E.; Roldan, M. A.; Wei, N. N.; Liu, Z. Q.; Martin, R. W.; Roqan, I. S. Generated Carrier Dynamics in V-Pit-Enhanced InGaN/GaN Light-Emitting Diode. ACS Photonics 2018, 5, 820-826.

(46) Miller, R. C.; Kleinman, D. A.; Nordland, W. A.; Gossard, A. C. Luminescence Studies of Optically Pumped Quantum Wells in GaasAlxga1-Xas Multilayer Structures. Phys. Rev. B: Condens. Matter Mater. Phys. 1980, 22, 863-871.

(47) Cheng, B.; Li, T.-Y.; Maity, P.; Wei, P.-C.; Nordlund, D.; Ho, K.-T.; Lien, D.-H.; Lin, C.-H.; Liang, R.-Z.; Miao, X.; Ajia, I. A.; Yin, J.; Sokaras, D.; Javey, A.; Roqan, I. S.; Mohammed, O. F.; He, J.-H. Extremely Reduced Dielectric Confinement in Two-Dimensional 
Hybrid Perovskites with Large Polar Organics. Commun. Phys. 2018, $1,80$.

(48) Gurioli, M.; Vinattieri, A.; Colocci, M.; Deparis, C.; Massies, J.; Neu, G.; Bosacchi, A.; Franchi, S. Temperature-Dependence of the Radiative and Nonradiative Recombination Time in Gaas/Alxga1-Xas Quantum-Well Structures. Phys. Rev. B: Condens. Matter Mater. Phys. 1991, 44, 3115-3124.

(49) Muth, J. F.; Lee, J. H.; Shmagin, I. K.; Kolbas, R. M., Jr.; Casey, H. C.; Keller, B. P.; Mishra, U. K.; DenBaars, S. P. Absorption coefficient, energy gap, exciton binding energy, and recombination lifetime of GaN obtained from transmission measurements. Appl. Phys. Lett. 1997, 71, 2572-2574.

(50) Nippert, F.; Mazraehno, M. T.; Davies, M. J.; Hoffmann, M. P.; Lugauer, H.-J.; Kure, T.; Kneissl, M.; Hoffmann, A.; Wagner, M. R. Auger recombination in AlGaN quantum wells for UV light-emitting diodes. Appl. Phys. Lett. 2018, 113, No. 071107.

(51) Dai, Q.; Schubert, M. F.; Kim, M. H.; Kim, J. K.; Schubert, E. F.; Koleske, D. D.; Crawford, M. H.; Lee, S. R.; Fischer, A. J.; Thaler, G.; Banas, M. A. Internal quantum efficiency and nonradiative recombination coefficient of $\mathrm{GaInN} / \mathrm{GaN}$ multiple quantum wells with different dislocation densities. Appl. Phys. Lett. 2009, 94, 111109. (52) Dai, Q.; Shan, Q. F.; Cho, J.; Schubert, E. F.; Crawford, M. H.; Koleske, D. D.; Kim, M. H.; Park, Y. On the symmetry of efficiencyversus-carrier-concentration curves in GaInN/GaN light-emitting diodes and relation to droop-causing mechanisms. Appl. Phys. Lett. 2011, 98, No. 033506.

(53) Alwadai, N.; Ajia, I. A.; Janjua, B.; Flemban, T. H.; Mitra, S.; Wehbe, N.; Wei, N. N.; Lopatin, S.; Ooi, B. S.; Roqan, I. S. CatalystFree Vertical ZnO-Nanotube Array Grown on p-GaN for UV-LightEmitting Devices. ACS Appl. Mater. Interfaces 2019, 11, 2798927996.

(54) Peretti, J.; Weisbuch, C.; Iveland, J.; Piccardo, M.; Martinelli, L.; Speck, J. S. Identification of Auger Effect As the Dominant Mechanism for Efficiency Droop of LEDs; SPIE, 2014; Vol. 9003.

(55) Kioupakis, E.; Rinke, P.; Delaney, K. T.; Van de Walle, C. G. Indirect Auger recombination as a cause of efficiency droop in nitride light-emitting diodes. Appl. Phys. Lett. 2011, 98, 161107.

(56) Hammersley, S.; Watson-Parris, D.; Dawson, P.; Godfrey, M. J.; Badcock, T. J.; Kappers, M. J.; McAleese, C.; Oliver, R. A.; Humphreys, C. J. The consequences of high injected carrier densities on carrier localization and efficiency droop in InGaN/GaN quantum well structures. J. Appl. Phys. 2012, 111, No. 083512.

(57) Kim, M.-H.; Schubert, M. F.; Dai, Q.; Kim, J. K.; Schubert, E. F.; Piprek, J.; Park, Y. Origin of efficiency droop in GaN-based lightemitting diodes. Appl. Phys. Lett. 2007, 91, 183507.

(58) Bochkareva, N. I.; Voronenkov, V. V.; Gorbunov, R. I.; Zubrilov, A. S.; Lelikov, Y. S.; Latyshev, P. E.; Rebane, Y. T.; Tsyuk, A. I.; Shreter, Y. G. Defect-related tunneling mechanism of efficiency droop in III-nitride light-emitting diodes. Appl. Phys. Lett. 2010, 96, 133502.

(59) Pak, Y.; Park, W.; Mitra, S.; Sasikala Devi, A. A.; Loganathan, K.; Kumaresan, Y.; Kim, Y.; Cho, B.; Jung, G.-Y.; Hussain, M. M.; Roqan, I. S. Enhanced Performance of $\mathrm{MoS}_{2}$ Photodetectors by Inserting an ALD-Processed $\mathrm{TiO}_{2}$ Interlayer. Small 2018, 14, 1703176 .

(60) Hader, J.; Moloney, J. V.; Koch, S. W. Density-activated defect recombination as a possible explanation for the efficiency droop in GaN-based diodes. Appl. Phys. Lett. 2010, 96, 221106.

(61) Feng, Z. C. III-Nitride Devices and Nanoengineering; Imperial College Press: London, 2008; pp 66-68.

(62) Binder, M.; Nirschl, A.; Zeisel, R.; Hager, T.; Lugauer, H.-J.; Sabathil, M.; Bougeard, D.; Wagner, J.; Galler, B. Identification of nnp and npp Auger recombination as significant contributor to the efficiency droop in (GaIn)N quantum wells by visualization of hot carriers in photoluminescence. Appl. Phys. Lett. 2013, 103, No. 071108. 